\title{
HIV/aids e meia idade: avaliação do conhecimento de indivíduos da região do Vale do Sinos (RS), Brasil
}

\author{
HIV/aids and middle aged: knowledge assessment \\ of people from Vale do Sinos, Rio Grande do Sul State, Brazil
}

Alexandre Lazzarotto ${ }^{1}$ Marina Tonin Reichert ${ }^{1}$ Carolina Venker ${ }^{1}$ Andréa Sebben Kramer ${ }^{1}$ Eduardo Sprinz ${ }^{2}$
${ }^{1}$ Instituto de Ciências da Saúde, Centro Universitário Feevale. Campus II RS 239, 2755 , Vila N ova. 93352-000 Novo Hamburgo RS. alazzar@terra.com.br ${ }^{2}$ Serviço de M edicina Interna, Hospital de Clínicas de Porto Alegre.
Abstract The objective of this article is to assess the knowledge about HIV/aids in middle aged acquaintance group participants from the Vale do Sinos, Rio Grande do Sul State, Brazil. A prospective cross-sectional study with 168 individuals (9.5\% maleand $90.5 \%$ female) between 40 and 59 years of age was performed. A questionnaire comprising questions about the concept, transmission, vulnerability, prevention, and treatment domains on HIV issues was constructed. It was observed that $61.3 \%$ of the sample had finished elementary education and $45.2 \%$ had a monthly incomeranging from 1 to 3 minimum wages. In the conceptual domain, $65.2 \%$ did not know that HIV infection has an asymptomatic phase and $34.5 \%$ believed HIV could be transmitted by a mosquito bite. In prevention and vulnerability domains, $19.5 \%$ had no knowledge about female condoms and $29.2 \%$ believed that the disease was confined to specific groups. Regarding to antiretroviral treatment, $12.5 \%$ had no idea about its existence. According to findings, middleaged participantsfrom acquaintance groups in Vale do Sinos had misconceptions about HIV/aids which might enhance the risk of infection. Therefore it is necessary to provide public health programs directed to this population group in order to prevent or decrease the risk of HIV transmission.

Key words HIV/aids, M iddle aged, Knowledge
Resumo 0 objetivo deste estudo é avaliar o nível de conhecimento sobre HIV/aids nos indivíduos de meia idade, participantes de grupos de convivência do Vale do Sinos (RS). 0 estudo caracterizou- se como transversal, obtendo-se uma amostra de 168 indivíduos, sendo 9,5\% homens e 90,5\% mulheres, na faixa etária entre 40 e 59 anos. Utilizou-se como instrumento um questionário composto pelos domínios "conceito", "transmissão", "vulnerabilidade", "prevenção" e"tratamento". 0 ensino fundamental caracterizou $61,3 \%$ da escolaridadedos participantes ea renda mensal situouse entre um e três salários mínimos para 45,2\%. No domínio "conceito", 65,2\% desconheciam a fase assintomática da infecção e 34,5\% apontaram o mosquito como transmissor da aids, no domínio "transmissão". Nos domínios "prevenção" e "vulnerabilidade", 19,5\% desconheciam a existência do preservativo feminino e $29,2 \%$ acreditaram que a doença é característica de grupos específicos. Quanto ao "tratamento", 12,5\% ignoraram a sua existência. De acordo com os achados, os indivíduos da meia idadeparticipantes degrupos deconvivência do Vale do Sinos tinham conceitos errôneos sobre HIV/aids que poderiam aumentar 0 risco à infecção. Sendo assim, é necessário proporcionar programas de saúde pública direcionados para esta população para prevenir ou diminuir o risco de transmissão do HIV.

Palavras-chave HIV/aids, M eia idade, Conhecimento 
Introdução

A aids é a manifestação clínica avançada da infecção pelo vírus da imunodeficiência humana (HIV) e caracteriza-se pela depleção das células com marcador fenotípico CD4+, principalmente os linfócitos $T$, o que leva à imunodepressão e ao consequente desenvolvimento de doenças oportunistas e outras complicações, tais como complicações no sistema nervoso central. A via sexual constitui a principal forma de transmissão do HIV, representando entre $75 \%$ e $85 \%$ do total de casos de aids no mundo ${ }^{1,2}$.

A utilização de práticas de proteção em relação às infecções sexualmente adquiridas (IST) e, principalmente, à aids, envolve questões como sexo, classe social, etnia e faixa etária. A variável idadetem grandeimportância nesteaspecto, tendo em vista a participação ativa de pessoas mais velhas na sociedade, principalmente na fai xa etária demeia idade (definidano Brasil entre 40 e 59 anos $^{3}$ ), como consequência do aumento da expectativa de vida na população ${ }^{4}$.

Desdeos primeiros casos registrados em 1981, já foram identificados 433 mil casos da aids no Brasil e, atualmente, destaca-se um aumento na incidência da infecção pelo HIV em indivíduos de meia idade em relação às pessoas com menos de quarenta an $05^{5,6}$ : Entre 1996 e 2005, a taxa de incidência entre os homens, na faixa etária de 50 a 59 anos, passou de 18,2 para 29,8 e, entre as mulheres, de 6,0 para $17,35,7$. Os casos de aids na meia idade atéjunho de 2006 total izavam 112.059 , sendo 78.299 homense 33.760 mulheres (Gráfico 1)7.
O Rio Grande do Sul é o terceiro estado brasileiro que apresenta maior número de casos de aids no Brasil7. Segundo o M inistério da Saúde, até o primeiro semestre de 2006, foram diagnosticados 10.188 casos de aids em indivíduos de meia idade no Rio Grande do Sul ${ }^{8}$. A região do Vale do Sinos merece destaque pois verificou-se que a epidemia está ocorrendo entre municípios pequenos, não se restringindo apenas aos centros urbanos ${ }^{9}$. Na cidade de Novo Hamburgo (pertencenteà zona metropolitana de Porto Alegre), por exemplo, foi observado que $44,8 \%$ dos casos abrangeram indivíduos de meia idade, somente na primei ra metade de $2006^{8}$.

Osaltosíndices deinfecção pelo HIV seguem em crescimento em todo o território nacional, atribuídos aos avanços tecnológicos na área da saúde, dentre eles a reposição hormonal, medicamentos para disfunção erétil, injeções e próteses, o que têm prolongado a vida sexual do ser humano, além da desmistificação do sexo, que vem tornando os indivíduos de meia idade mais conscientes quea sexualidade podeser partevital durante esses anos ( 40 a 59) e também em anos posteriores ${ }^{3,10,11}$.

É difícil determinar taxas de infecção pelo HIV entre os indivíduos de meia idade, pelo fato de poucos indivíduos com mais de cinquenta e que se exponham a alguma situação de risco para a infecção pelo HIV realizarem o teste rotineiramente. Outros aspectos relevantes são os sintomas da infecção; por serem inespecíficos, podem ser atribuídos às doenças comuns do envelhecimento, tais como doenças respiratórias, caque-

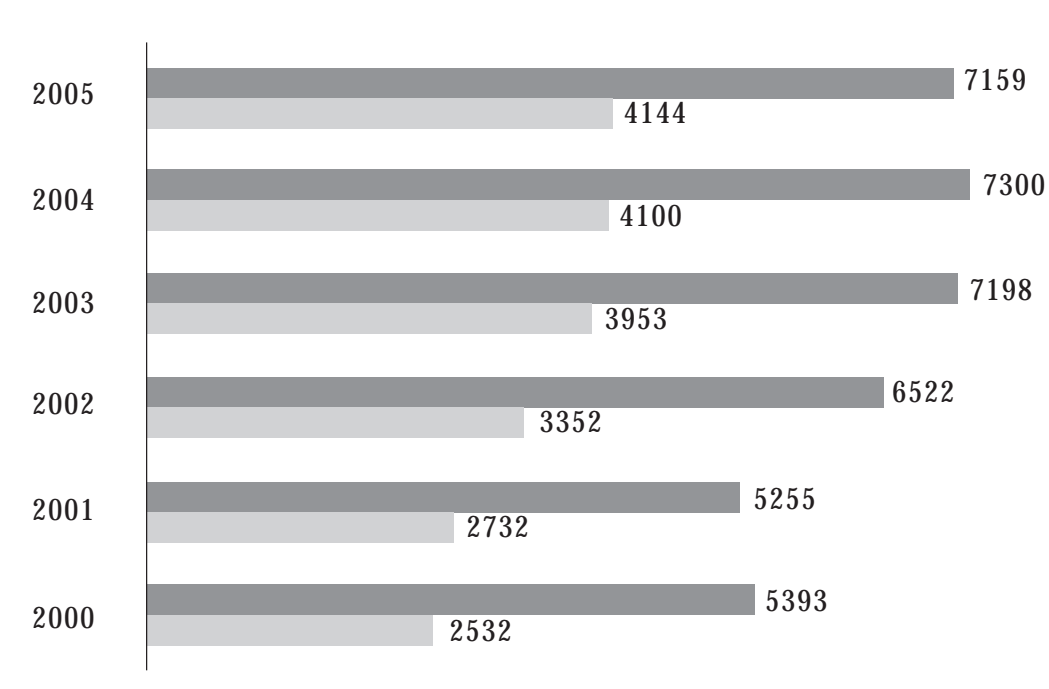

Homens

Mulheres 
xia, problemas de memória, entre outras. Sendo assim, infelizmente, a maioria dosindivíduosmais velhos são diagnosticados HIV positivos somente no estágio tardio da infecção $0^{12-14}$.

É de suma importância conhecer a perspectiva cognitiva de diferentes faixas etárias e contextos culturais para, então, realizar-se práticas que possam elucidar questões relacionadas à infecção e diminuir o preconceito em relação aos portadores do HIV. Torna-se necessário conhecer o comportamento sexual e as condições de vida da população para analisar as necessidades e contribuir para a preven ção de novos casos de aids ${ }^{4}$. Nesta perspectiva, cita-se o estudo de Berquó et al. ${ }^{15}$, que avaliaram o comportamento sexual da população brasileira e as percepções sobre HIV/ aids de indivíduos na faixa etária de 16 a 65 anos, residentes em áreas urbanas de 169 microrregiões, evidenciando falta deinformação nas questões sobre a retirada do pênis antes da relação sexual $(40,9 \%)$, uso dos preservativos masculino $(31,3 \%)$ e feminino $(45,6 \%)$, banheiro público $(37,2 \%)$ e uso de agulhas e seringas utilizadas $(40,5 \%)$. 0 estudo de Brasileiro et al. ${ }^{16}$, realizado com indivíduos HIV positivosinternados em um hospital em Goiás, corrobora a afirmação anterior, pois constatou grande carência de conhecimento, referindo-se à qualidade de vida e ao enfrentamento da doença, tanto dos pacientes quanto da população em geral e profissionais.

A partir das premissas anteriores, há necessidade deum trabalho de educação em saúde, agregando profissionais envolvidos nesta temática e a população $0^{16}$. Observa-se uma carência de trabalhos na literatura sobre o conhecimento e a abordagem da aids no contexto da meia idade ${ }^{17}$. A partir desta carência, este estudo tem como objetivo principal identificar o conhecimento de HIV/aids dos indivíduos de meia idade, participantes dos grupos de convivência do Vale do Sinos, Rio Grande do Sul, Brasil.

\section{Métodos}

0 estudo caracterizou-se como transversal, sendo a amostra composta pelos integrantes dos grupos de convivência da terceira idade do Vale do Sinos ea participação realizada pelo consentimento informado.

Para obtenção dos dados, utilizou-seum instrumento validado que abrangia características gerais como nível socioeconômico, idade, escolaridade, presença de companheiro(a) ereligião. o questionário é composto de treze questões fe chadas (com opções de verdadeiro, falso e não sei ), organizadas nos domínios "conceito", "transmissão", "vulnerabilidade", "prevenção", e "tratamento". Foram abordadas também questões específicas, que incluíram aids como um castigo divino, o conhecimento de portadores do HIV pelo participante, a utilização de preservativos e a realização de teste anti-HIV.

Os dados foram coletados de agosto de 2005 a maio de 2006, em 47 grupos de convivência da terceira idade, pertencentes às catorze cidades da Região do Vale do Sinos (Araricá, Campo Bom, Canoas, DoisIrmãos, Estância Velha, Esteio, Ivoti, Nova Hartz, N ova Santa Rita, N ovo Hamburgo, Portão, São Leopoldo, Sapiranga, Sapucaia do Sul), classificadas de acordo com o Conselho Regional de Desenvolvimento (CORE$D E S)^{18}$. A idade mínima de ingresso nos grupos de convivência era quarenta anos, situação que permitiu a realização da pesquisa em indivíduos de meia idade.

Inicialmente, os pesquisadores identificaram os grupos convivência do Vale do Sinos e seus coordenadores para, posteriormente, explicarIhes a pesquisa e convidar os seus integrantes a participarem dela. Havendo 0 aceite do coordenador (a), agendava-se com ele (a) um encontro no dia de reunião do grupo para explicar todos os procedimentos da pesquisa, esclarecer as dúvidas e, após, convidar todos integrantes presentes a responder o questionário, salientando que a participação era voluntária e que ocorreria 0 retorno dos pesquisadores ao grupo para a apresentação dos resultados na forma de oficinas.

A pós o término da pesquisa e da identificação das lacunas nos domínios estudados, houve o retorno dos resultados aos participantes dos grupos estudados e outros grupos interessados na temática. A dinâmica desteretorno foi através do desenvolvimento de oficinas específicas para o contexto de grupos convivência, utilizando-se recurso multimídia, folder individual, cartazes e preservativos masculino efeminino.

\section{Resultados}

A amostra foi composta por 168 indivíduos, sendo 9,5\% (16) homens e 90,5\% mulheres (152), na faixa etária entre 40 e 59 anos, com idade média e desvio padrão de 54 anos $\pm 4,6$. 0 ensino fundamental incompleto caracterizou $61,3 \%$ da escolaridade dos participantes. Considerando a religião, $76,2 \%$ dosindivíduos eram católicos. Em relação à presença de companheiro, 62,5\% rela- 
tou possuir companheiro (a). 0 nível socioeconômico da maioria dos participantes $(45,2 \%)$ estabeleceu-se entre um e três salários mínimos (Tabela 1).

Do total pesquisado, 38,7\% relataram que conheciam algum portador (a) do HIV, 23,2\% já haviam realizado o testeanti-HIV, 64,3\% não utilizavam preservativo nas relações sexuais e $34,5 \%$ consideram a aids como um castigo divino para aqueles que cometeram pecados. As questões relacionadas à aids estão descritas na Tabela 2.

\section{Discussão}

No domínio "conceito", a maioria dos participantes desconhecia a fase assintomática da aids, visto que $67,2 \%$ dos indivíduos erraram ou não sabiam responder a questão. 0 período entre a aquisição do HIV ea manifestação da aids podedurar em média de oito a dez anos ${ }^{19}$. Neste período, 0 indivíduo, apesar denão apresentar complicações associadas à infecção, continua sendo transmissor do HIV ${ }^{20}$. Desta forma, o desconhecimento

Tabela 1. Características gerais dos participantes da pesquisa $(n=168)$.

\begin{tabular}{|c|c|c|}
\hline Características & $\%$ & Frequência \\
\hline Sexo & 9,5 & 16 \\
\hline Masculino & 90,5 & 152 \\
\hline \multicolumn{3}{|l|}{ Feminino } \\
\hline Idade & 16,0 & 27 \\
\hline 40 a 49 anos & 84,0 & 141 \\
\hline \multicolumn{3}{|l|}{50 a 59 anos } \\
\hline Escolaridade & 3,6 & 6 \\
\hline Nenhuma & 16,7 & 28 \\
\hline 1 a 3 anos & 44,6 & 75 \\
\hline 4 a 7 anos & 35,1 & 59 \\
\hline \multicolumn{3}{|l|}{8 ou mais anos } \\
\hline Renda mensal * & 28,6 & 48 \\
\hline Até 1 salário mínimo & 45,2 & 76 \\
\hline 1 a 3 salários mínimos & 14,9 & 25 \\
\hline 4 a 6 salários mínimos & 7,8 & 13 \\
\hline \multicolumn{3}{|l|}{ M ais de 7 salários mínimos } \\
\hline Religião ** & 76,2 & 128 \\
\hline Católica & 14,3 & 24 \\
\hline Evangélica & 4,2 & 7 \\
\hline \multicolumn{3}{|l|}{ Outras } \\
\hline Companheiro (a) & 63,7 & 107 \\
\hline $\mathrm{Sim}$ & 36,3 & 61 \\
\hline Não & & \\
\hline
\end{tabular}

*3,6\% (6) não informaram a resposta; ** $5,4 \%$ (9) não informaram a resposta. desta fase, aliada à fal ta de proteção durante 0 ato sexual, coloca os indivíduos em posição vulnerável para a transmissão do vírus s21,22. $^{2}$

No domínio "transmissão", quando indagados se o mosquito poderia ser o transmissor da aids, metade dos participantes apresentaram dúvidas, pois 34,5\% dos participantes erraram a questão e 15,5\% não sabiam respondêla. É evidente que a transmissão por picada de insetos não está incluída na epidemiologia da aids ${ }^{23,24}$. As células dos artrópodes não possuem o antígeno $T_{4}$, necessário para a replicação do vírus. $\mathrm{A}$ baixa infectividadee a sobrevivência do vírus por um curto período fora do organismo humano evidenciam a inexistência desta possibilidade ${ }^{25}$. Os estudos de Fernandes ${ }^{26}$, realizados em uma favela do Rio de Janeiro com sujeitos de 13 a 49 anos de idade e em quatro capitais brasileiras, evidenciaram que, respectivamente, $41,1 \%$ e $52 \%$ dos participantes acreditavam ser a picada de mosquito uma forma de transmissão do HIV. Reitera-se que as principais vias de contaminação são a sexual, a parenteral e a vertical ${ }^{13,23}$.

No domínio "prevenção", questões como a identificação do preservativo como forma de prevenção à infecção ea existência de uma camisinha específica para mulheres obtiveram alta porcentagem de acerto entre os participantes; porém, quando questionados sobre o uso de preservativo nas relações sexuais, $64,3 \%$ não 0 utilizavam. Tais indivíduos podem não considerar o uso do preservativo como uma necessidade, especialmente mulheres que estão na menopausa e que não precisam preocupar-se em prevenir uma gravidez, pois associam o preservativo apenas a um método contraceptivo. À medida que as mulheres envelhecem, ocorrem algumas mudanças orgânicas, tornando-as mais vulneráveis às lesões durante as relações sexuais ${ }^{17}$, além de cultuarem o pensamento de que a situação de ter um parceiro fixo exclui a utilidade do preservativo ${ }^{6}$.

As restrições em relação à sexualidade nas décadas passadas evidenciam o motivo da resistência dos indivíduos mais vel hos à educação sexual, principalmente ao fato do uso de preservativos, pois as gerações mais antigas excluíram esta população do conhecimento sobre o HIV/ aids, em razão da falta de estratégias apropriadas para orientar as pessoas, pois a sexualidade era pouco abordada no contexto familiar ${ }^{3}$.

Há muito tempo não se considera grupos de risco e sim situações de risco, nas quais qualquer indivíduo pode estar exposto à infecção pelo HIV; entretanto, na faixa etária estudada, ainda secon- 
Tabela 2. Conhecimentos gerais sobre a aids dos participantes do estudo.

\begin{tabular}{|c|c|c|c|}
\hline Domínios (n=168) & Acertos & Erros & Não sabia \\
\hline \multicolumn{4}{|l|}{ Conceito } \\
\hline O vírus HIV é o causador da aids & $139(82,7 \%)$ & $6(3,6 \%)$ & $23(13,7 \%)$ \\
\hline $\begin{array}{l}\text { A pessoa com o vírus da aids sempre apresenta os sintomas } \\
\text { da doença }\end{array}$ & $55(32,7 \%)$ & $76(45,2 \%)$ & $37(22 \%)$ \\
\hline $\begin{array}{l}0 \text { vírus da aids é identificado através de exames de } \\
\text { laboratório }\end{array}$ & $158(94 \%)$ & $7(4,2 \%)$ & $3(1,8 \%)$ \\
\hline \multicolumn{4}{|l|}{ Transmissão } \\
\hline $\begin{array}{l}\text { O vírus da aids pode ser transmitido por sabonetes, toalhas } \\
\text { e assentos sanitários }\end{array}$ & $128(76,2 \%)$ & $23(13,7 \%)$ & $17(10,1 \%)$ \\
\hline $\begin{array}{l}0 \text { vírus da aids pode ser transmitido por abraço, beijo no } \\
\text { rosto, beber no mesmo copo e chimarrão }\end{array}$ & $133(79,2 \%)$ & $26(15,4 \%)$ & $9(5,4 \%)$ \\
\hline O vírus da aids pode ser transmitido por picada de mosquito & $84(50 \%)$ & $58(34,5 \%)$ & $26(15,5 \%)$ \\
\hline \multicolumn{4}{|l|}{ Prevenção } \\
\hline $\begin{array}{l}\text { A pessoa que usa camisinha nas relações sexuais impede a } \\
\text { transmissão do vírus da aids }\end{array}$ & $145(86,3 \%)$ & $18(10,7 \%)$ & $5(3,0 \%)$ \\
\hline Existe uma camisinha específica para as mulheres & $137(81,5) \%$ & $8(4,8 \%)$ & $23(13,7 \%)$ \\
\hline $\begin{array}{l}\text { O uso da mesma seringa e agulha por diversas pessoas } \\
\text { transmite aids }\end{array}$ & $162(96,4 \%)$ & $3(1,8 \%)$ & $3(1,8 \%)$ \\
\hline \multicolumn{4}{|l|}{ Vulnerabilidade } \\
\hline $\begin{array}{l}\text { A aids é uma doença que ocorre somente em homossexuais } \\
\text { masculinos, prostitutas (os) e usuários (as) de drogas }\end{array}$ & $110(65,5 \%)$ & $49(29,1 \%)$ & $9(5,4 \%)$ \\
\hline $\begin{array}{l}\text { Os indivíduos da terceira idade não devem se preocupar } \\
\text { com a aids, pois ela atinge apenas os jovens }\end{array}$ & $142(84,5 \%)$ & $22(13,1 \%)$ & $4(2,4 \%)$ \\
\hline \multicolumn{4}{|l|}{ Tratamento } \\
\hline A aids é uma doença que tem tratamento * & $129(76,8 \%)$ & $21(12,5 \%)$ & $17(10,1 \%)$ \\
\hline A aids é uma doença que tem cura & $124(73,8 \%)$ & $18(10,7 \%)$ & $26(15,5 \%)$ \\
\hline
\end{tabular}

${ }^{*} 1(0,6 \%)$ não informou a resposta.

cebe a idéia de que a aids é uma característica específica de homens que fazem sexo com homens, profissionais do sexo e narcótico dependentes ${ }^{23}$, pois aproximadamente $29 \%$ participantes a consideraram característica de grupos específicos. A partir da transmissão heterossexual, houveuma mudança no perfil da epidemia, constituindo-seatualmenteesta transmissão em uma característica importante para o aumento do número de casos em mulheres 9 .

Quanto ao domínio "tratamento", 22,6\% dos participantes desconheciam ou não sabiam sobrea existência de tratamento para a doença. De fato, a aids constitui-se uma doença sem cura; no entanto, existe tratamento através dos antirretrovirais. A terapia antirretroviral combinada (TARV) é potencialmente eficaz na redução da carga viral plasmática, tornando mais lenta a progressão da doença ${ }^{27}$. Os medicamentos que compõem a terapia são divididos em classes: inibidores de transcriptase reversa, análogos e não análogos denucleotídeo, inibidores de proteasee inibidores de fusão. 0 tratamento é disponibilizado pela Secretaria da Saúde, com distribuição de medicamentos nos Serviços deAssistência Especializada (SAE) dos municípios ${ }^{28}$.

\section{Conclusão}

A partir da análise dos dados da pesquisa, foram evidenciadas lacunas no conhecimento sobre HIV/aids pela população de meia idade e que devem ser preenchidas, visando ao declínio de novos casos de aids com a adoção de medidas de prevenção e, assim, a melhoria na qualidade de vida desta população, além da diminuição do preconceito com os portadores do HIV.

Torna-se necessária a implantação de programas de educação para a saúde que proporcionem 0 acesso mais facilitado às informações sobre a infecção pelo HIV para a população em 
geral, concomitanteà ampliação do sestudos nos diversos aspectos relacionados ao HIV/aids na população de meia idade.

\section{Colaboradores}

AR Lazzarotto é autor da pesquisa e trabalhou na elaboração e redação final do artigo. AS K ramer trabal hou na redação do artigo. M Reichert e C Venker trabalharam na pesquisa e redação do artigo. E Sprinz trabalhou na redação final.

\section{Referências}

1. Abbas AK, Lichtman AH, Pober JS. Cellular and molecular immunology. Philadelphia: W.B. Saunders Company; 2000.

2. Lopes MV, Fraga MN. Pessoas vivendo com HIV: estresse e suas formas de enfrentamento. Rev Latino-am Enfermagem 1998; 6(4):75-81.

3. Papalia DE, Olds SW. Desenvolvimento humano. 7ạ ed. Porto Alegre: Artmed; 2000.

4. Ferreira MP. Knowledge and risk perception of HIV/ aids: a profile of the Brazilian people in 1998. Cad Saude Publica 2003; 19(2):213-222.

5. Centers for Disease Control and Prevention. The Global HIV/aids Pandemic, 2006. M MWR 2006; 18(55):881.

6. De Carlo P, Linsk N. What are HIV prevention needs of adults over 50? San Francisco: University of California/Center for Aids Prevention Studies; 1997.

7. Brasil. Ministério da Saúde. Boletim Epidemiológico DST/aids 2006. [site da Internet] [acessado 2006 nov 29]. Disponível em: http://www.aids.gov.br

8. Brasil. M inistério da Saúde. Tabulação de dados de HIV/aids no Rio Grande do Sul. [site da Internet] [acessado 2007 mar 15]. Disponível em: http:// www.aids.gov.br/cgi/tabcgi.exe?tabnet/rs.def

9. De Brito AM, Castilho EA, Szwarcwald CL. Aids e infecção pelo HIV: uma epidemia multifacetada. Rev. Soc. Bras. M ed. Trop. 2001; 34(2):207-217.

10. Soares AM, Lima WJR, M arrochi LCR, Silveira CM . Aids no idoso. In: Freitas EV, Py L, Néri AL, Cançado FAX, Gorzoni ML, Rocha SM, organizadores. Tratado de geriatria e gerontologia. Rio de Janeiro: Guanabara Koogan; 2002.

11. Lazzarotto A, Kramer AS, Hadrich M, Tonin M Caputo P, Sprinz E. O conhecimento de HIV/aids na terceira idade: estudo epidemiológico no Vale do Sinos, Rio Grande do Sul, Brasil. Cien Saude Colet [periódico na Internet] [acessado 2007 mai]. Disponível em: http://www.abrasco.org.br/cienciaesaude coletiva/artigos/artigo_int.php?id_artigo $=644$

12. Kohli R, Klein RS, Schoenbaum EE, Anastos K, Minkoff H, Sacks HS. Aging and HIV Infection. J U rban Health 2006; 83(1):31-42.

13. Inelmen EM, Gasparini G, Enzi G. HIV/aids in older adults: A case report and literature review. Infectious Disease 2005; 60(9):26-30.

14. Gebo KA. HIV and Aging: Implications for Patient $M$ anagement. Drugs Aging 2006; 23(11):897-913.

15. Berquó E, Souza MR, Pinho M D G, Bussab W, Loyola MA, Ferreira MP, Correa M. Comportamento se xual da população brasileira e percepções sobre HIV/ Aids [relatório final de pesquisa]. Brasília: Ministério da Saúde/Centro Brasileiro de Análise e Planejamento; 1999.
16. Brasileiro M, Freitas MI. Representações sociais sobre aids de pessoas acima de 50 anos de idade, infectadas pelo HIV. Rev Latino-am Enfermagem 2006; 14(5):789-795.

17. Pogrányivá AH. El sida y lãs personas mayores. Gerokomos 2006; 17(2):82-88.

18. Conselho Regional de Desenvolvimento. [site da Internet] [acessado 2005 mai 23]. Disponível em: http://www.fee.rs.gov.br/sitefee/pt/content/resumo/ pg coredes.php

19. Canini SR, Reis RB, Pereira LA, Gir E, Pelá NTR. Qualidade de vida de indivíduos com HIV/aids: uma revisão de literatura. Rev Latino-am Enfermagem 2004; 12(6):940-945.

20. Parham P. 0 sistema imune. Porto Alegre: Artmed; 2001.

21. Vergara TRC, Barroso PF. Transmissão Sexual do HIV. Tendências em HIV/Aids [periódico na Internet] 2007 [acessado 2008 mar 26];1(4):[cerca de 8 p.]. Disponível em: http://www.aids.gov.br

22. Marks G, Burris S, Peterman T. Reducing sexual transmission of HIV from those who know they are infected: the need for personal and collective responsibility. Aids 1999; 13:297-306.

23. Duncan BB, Schmidt MI, Giugliani E. M edicina ambulatorial: condutas de atenção primária baseada em evidências. 3ạ ed. Porto Alegre: Artmed; 2004.

24. Zuckerman A). Aids and insects. Br. Med J (Clin Res Ed) 1986; 6528:1094-1095.

25. Iqbal MM. Can we get aids from mosquito bites? J La State M ed Soc 1999; 151:429-433.

26. Fernandes JC. Evolução dos conhecimentos, atitudes e práticas relativas ao HIV/aids em uma população de favela do Rio de Janeiro. Cad Saude Publica 1998; 14(3):575-581.

27. Colombrini MRC, Lopes MHBM, Figueiredo RM . Adesão à terapia antiretroviral para a aids. Rev. Esc. Enf. USP 2006; 40(4):576-581.

28. Brasil. Ministério da Saúde. Tratamento de HIV e aids - M edicamentos. [site da Internet] [acessado 2006 dez 17]. Disponível em: http://www.aids.gov.br/ data/Pages/LU M ISIEE683AEPTBRIE.htm

Artigo apresentado em 10/09/2007

Aprovado em 30/01/2008

Versão final apresentada em 31/03/2008 